\title{
Pädagogik als Gefahrenzone
}

\author{
Vertrauen, Verletzbarkeit und Verantwortung als Erkenntniskategorien für \\ Aufarbeitung sexueller Gewalt in pädagogischen Kontexten
}

\author{
Sabine Andresen, Andrea Pohling, Nina Schaumann
}

\section{Pädagogik zwischen Vertrauen und Verletzbarkeit - zur Einleitung}

Zum Kindsein gehört es, Erwachsenen Vertrauen schenken zu müssen. ${ }^{1}$ In diesem Sinne bezeichnet der Bildungsphilosoph Roland Reichenbach (2011) Vertrauen als eine „Gabe“, durch die die Beziehung zwischen Kindern und Erwachsenen geprägt ist und über die sich Erziehung und Bildung vermittelt. Für das Kind, das Vertrauen schenkt, ergibt sich allerdings das prinzipielle Risiko, verletzt zu werden (Herzog 2013). Dies gilt auch für andere Altersphasen, gleichwohl ist das Vertrauen des Kindes in Erwachsene aufgrund von Ungleichheit - nicht nur der sozialen Positionen - besonders anfällig für Verletzbarkeit (Baier 2001). Angelehnt an diese Lesart wird im Folgenden Vertrauen als Schlüsselthema für die Erfahrungen von Betroffenen sexueller Gewalt in ihrer Kindheit und Jugend untersucht. ${ }^{2}$ Die Dynamik von Vertrauen und Verletzbarkeit ist zudem eine Perspektive für die gesellschaftliche Aufarbeitung sexueller Gewalt in pädagogischen Institutionen. Hier kommt insbesondere Verantwortung als normativer Bezugspunkt zum Tragen.

1 Der Beitrag ist im Rahmen des folgenden, vom Bundesministerium für Bildung und Forschung (BMBF) geförderten Verbundprojektes entstanden: „Auf-Wirkung: Aufarbeitung für wirksame Schutzkonzepte in Gegenwart und Zukunft. Erfahrungen von Betroffenen sexualisierter Gewalt und ihre Auswertung für Schutz in Institutionen, Leitlinien für Aufarbeitungs- und Schutzkonzepte“ (Projektkennzeichen: oıSRı7o9, Projektträger: Deutsche Luft- und Raumfahrt, DLR), Verbundkoordination: Sabine Andresen, GoetheUniversität Frankfurt, Teilprojekte: FIVE - Forschungs- und Innovationsverbund an der Evangelischen Hochschule Freiburg e.V., Sozialwissenschaftliches FrauenForschungsInstitut Freiburg - Berlin, Barbara Kavemann; Universitätsklinik Hamburg-Eppendorf, Peer Briken; Verein für psychosoziale Initiativen (VfPI) e.V., Institut für Praxisforschung und Projektberatung, Heiner Keupp; Universität Rostock, Jens Brachmann, einzusehen unter https:// www.empirische-bildungsforschung-bmbf.de/de/2378.php, zuletzt aufgerufen am 23.2.2021. Wir danken Johanna Christ und Lia Pollmann, beide studentische Hilfskräfte in Frankfurt, für ihre wertvollen Kommentare und Hinweise.

2 In diesem Text wird von sexualisierter oder sexueller Gewalt gesprochen. Dem liegt ein weiter Gewaltbegriff zugrunde, der sowohl sogenannte „Hands-Off“-Taten wie das Zeigen von Missbrauchsdarstellungen als auch „Hands-On“-Taten wie das Anfassen von Geschlechtsteilen des Kindes, Erzwingen, Vergewaltigung einbezieht (Jud 2014). 
Auch in einer erziehungswissenschaftlichen Perspektive ist dies relevant, weil die Person, der Vertrauen entgegengebracht oder geschenkt wird, in einer besonderen Verantwortung steht (Reichenbach 2011). Was für das Kind gegenüber Erwachsenen in einem generationalen Erziehungsverhältnis gilt, ist auch für erwachsene Betroffene manifest, wenn sie auf den Aufklärungsund Aufarbeitungswillen vertrauen und damit Familienangehörigen oder Vertreter:innen in Institutionen Verantwortung zukommt.

Während der interdisziplinären Tagung „Katholische Dunkelräume“ im Oktober 2020 ging es auf einem Panel „Missbrauch in Gesellschaft und Kirche. Bedingungsfaktoren in der Geschichte von Psychologie, Pädagogik und Recht" auch um Pädagogik. ${ }^{3}$ Dabei kamen katholische Schulen in den Blick sowie erziehungswissenschaftlich orientierte Befunde aus der Aufarbeitung sexuellen Kindesmissbrauchs. Der Vortrag identifizierte Vertrauen als eine kindheitstheoretische und pädagogische Schlüsselkategorie für die Klärung des Gewaltverständnisses, weil sexuelle Gewalt häufig im vertrauten Umfeld stattfindet, Betroffene das zerstörte Vertrauen des einstigen Kindes thematisieren und Vertrauen benötigen, um über Unrecht und Leid sowie den Umgang mit ihnen als Opfer bzw. Betroffene sprechen zu können. Vertrauen ist auch für Fragen der Aufklärung und Aufarbeitung sexueller Gewalt in der katholischen Kirche wichtig, weil viele Gläubige das Vertrauen in ihre Kirche verlieren. Auf Vortrag und Diskussion baut der vorliegende Beitrag auf und hat im Anschluss an die Tagung eine vertiefte Analyse schriftlicher Berichte an die Unabhängige Kommission zur Aufarbeitung sexuellen Kindesmissbrauchs vorgenommen (Unabhängige Kommission 2019a und b). In diesen Berichten erzählen Betroffene von Gewalterfahrungen, den Umständen ihrer Kindheit, den durch sexuelle Gewalt mit ausgelösten Ängsten, Schmerzen, Gefühlen sowie von Reaktionen aus dem nahen Umfeld. Die Berichte sind von ihrer Aufbereitung und Gestaltung her sehr unterschiedlich. Manche umfassen etwa vier bis fünf Seiten, einige enthalten nur wenige Sätze, andere sind umfangreiche Manuskripte, denen auch Schreiben an Behörden, Gedichte oder Zeichnungen beigefügt sind.

Im nächsten Abschnitt (2) soll in erziehungswissenschaftlicher Perspektive auf das Verhältnis von Vertrauen, Verletzbarkeit und Verantwortung eingegangen werden, um daran anschließend die Frage nach der Rolle der „Pädagogik als Gefahrenzone“ zu stellen (3). Im vierten Abschnitt wird die Unabhängige Kommission zur Aufarbeitung sexuellen Kindesmissbrauchs (4)

3 Titel des Vortrags „Pädagogik als Gefahrenzone. Erkenntnisse aus den Anhörungen Betroffener sexueller Gewalt in der Kindheit“, gehalten bei der interdisziplinären Tagung „Katholische Dunkelräume“ am 8.10.2020. 
vorgestellt und im fünften Abschnitt werden der Materialkorpus und die methodische Herangehensweise beschrieben (5). Dies führt im sechsten Abschnitt zur Darstellung ausgewählter Ergebnisse zu Vertrauen und Verletzbarkeit als Erkenntniskategorien auch für gesellschaftliche Aufarbeitung (6) und einem vorläufigen Resümee (7).

\section{Vertrauen, Verletzbarkeit und Verantwortung in einer erziehungswissenschaftlichen Perspektive}

Vertrauen in Erziehungsverhältnissen hat eine zweifache Dimension: die Abhängigkeit des Kindes, vertrauen zu müssen (Reichenbach 2011), sowie das Vertrauen der Eltern in andere Personen, denen sie ihr Kind anvertrauen (Bartmann/Pfaff/Welter 2012). ${ }^{4}$ Letzteres ist auch für die Aufarbeitung in der katholischen Kirche zentral, denn Eltern haben ihre Kinder als Messdiener, im Kommunionsunterricht, auf Jugendfreizeiten, aber auch in katholischen Schulen, Internaten und der Heimerziehung dem geistlichen und weltlichen Personal der Kirche anvertraut (Unabhängige Kommission 2019a, 162ff.; für Vertrauen und sexuelle Gewalt in der Heimerziehung s. Bütow 2012).

Darauf bezogen stellt sich die Frage, welche Bedeutung dem Phänomen zukommt, dass Kinder aufgrund ihres Alters und ihrer sozialen Position in besonderer Weise abhängig davon sind, anderen Menschen vertrauen zu müssen (Andresen 2020a). Erziehungsverhältnisse als stets auch durch Abhängigkeit geprägte Situationen geben darauf eine erste Antwort. So identifiziert der Gewaltforscher David Finkelhor (2008) den mangelnden Einfluss der Kinder auf Menschen, die sie betreuen, erziehen, pflegen, unterrichten als wesentliche Ursache für ihre Vulnerabilität. Hinzu kommt, dass stellvertretend für Kinder Entscheidungen getroffen werden (Brumlik 2017), und sie sich selbst kaum von ihrer Familie, den tradierten Erziehungs- und Gewaltvorstellungen oder vom sozioökonomischen Status ihrer Herkunft befreien können.

Angelehnt an die moralphilosophische Systematik Annette Baiers (2001) wird im Folgenden die für Erziehungsverhältnisse spezifische Dynamik zwischen Vertrauen, Verletzbarkeit und Verantwortung beschrieben. Baier sensibilisiert für kind- und erwachsenenspezifische Formen der Abhängigkeit, anderen vertrauen zu müssen. In einer historischen Annäherung differenziert

4 Wir reduzieren die systematische Diskussion schematisch auf das Verhältnis zwischen Kind und Erwachsenem bzw. zwischen einem Kind und seinen Eltern, wohl wissend, dass die Schattierungen dadurch wegfallen. 
sie zwischen Urvertrauen, Gottvertrauen (Glaube und Hoffnung), dem Vertrauen in Regierungen sowie dem Vertragsvertrauen, das in modernen Gesellschaften für maßgeblich gehalten werde. Sie weist nach, dass es in der Beziehung zwischen Kindern und Erwachsenen kein Vertragsvertrauen geben kann, weil dieses im Prinzip auf Gleichheit der Vertragspartner:innen beruhe. Im Verhältnis zum Kind aber dominiere eine unausweichliche Ungleichheit. Insofern sei das Vertrauen des Kindes eher dem Gottvertrauen ähnlich, und zwar als ein "angeborenes, zerbrechliches Vertrauen“ (ebd., 56). Kindliches Vertrauen bestehe darin, mächtigeren Personen Wohlwollen zuzuschreiben. Durch Erfahrungen im Prozess des Aufwachsens kommt es zu Veränderungen, die im Falle von Grenzverletzung und Gewalt zum existenziellen Vertrauensverlust führen können.

Ein anderer, wichtiger Aspekt kindlichen Vertrauens sei an dieser Stelle ebenfalls benannt. Kinder erhalten vor allem in Interaktion mit ihnen unmittelbar nahen Vertrauenspersonen die Chance, Selbstvertrauen auszubilden (Erikson 2005; Spitz 1976). Während bindungstheoretische Arbeiten sich auf Vertrauen in den Primärbeziehungen konzentrieren, also zwischen einem Kind und der ersten zentralen Bezugsperson - meistens werden Mutter-KindDynamiken untersucht - gehen erziehungswissenschaftliche Ansätze auf die Bedeutung von Vertrauen in professionellen Beziehungen zu Kindern und Jugendlichen ein (Fabel-Lama/Tiefel/Zeller 2012).

Die Person, der Vertrauen entgegengebracht wird, hat einen Ermessensspielraum, wie sie den Vertrauensvorschuss deuten und diesem gerecht werden kann. Dieser Spielraum und die Möglichkeiten der Deutung sind sowohl in familiären Nahebeziehungen als auch in pädagogischen Kontexten relevant, denn Kinder selbst haben darüber kaum eine Kontrolle. Ermessensspielräume bergen in Beziehungen, die auf Gleichheit beruhen, weniger Risiken als in asymmetrischen Erziehungs- und Sorgeverhältnissen. So kann es in einer auf Gleichheit beruhenden Beziehung zwischen selbständigen Erwachsenen beispielsweise genau richtig sein, sich des Vertrauens würdig zu erweisen, indem keine Einmischung in das Leben der sich anvertrauenden Person erfolgt. Dies würde jedoch in der Regel gegenüber einem Kind nicht ausreichen: „Die wichtigsten Dinge, die wir anderen anvertrauen, sind Dinge, die mehr brauchen um zu gedeihen, als Nichteinmischung." (Baier 2001, 48)

Der normative Bezugspunkt für den Ermessensspielraum gegenüber einem Kind ist, seinem Wohl möglichst gerecht zu werden. Besonders von sexueller Gewalt betroffene Kinder benötigen in der Regel anderes als eine Nichteinmischung. Doch gerade davon berichten viele Betroffene und legen Zeugnis über verweigerte Hilfe, ungläubige Abwendung und das Schweigen der anderen ab (Andresen 2020a). 


\section{Perspektiven auf „Pädagogik als Gefahrenzone“}

Wird das Vertrauen des Kindes nachhaltig missbraucht, so erweist sich Pädagogik durchaus als „Gefahrenzone“. Welche besonderen Aspekte - Stichwort „spiritueller Missbrauch“ (Wagner 2019) - sich daraus für eine Pädagogik unter dem Dach der katholischen Kirche ergeben, wurde in verschiedenen Beiträgen der Tagung aufgegriffen. Insbesondere das fehlende Einfühlungsvermögen in das Leid betroffener Kinder und Jugendlicher sticht im Kontext der katholischen Kirche hervor. Doch der Mangel an Betroffenenorientierung und Aufklärungsbereitschaft zeigt sich auch in anderen gesellschaftlichen Bereichen. In diesem Abschnitt geht darum, dass auch in der Erziehungswissenschaft Gewalt und vor allem sexualisierte Gewalt gegen Kinder und Jugendliche lange randständig behandelt wurden. Im „Handbuch sexualisierte Gewalt und pädagogische Kontexte" (Retkowski/Treibel/Tuider 2018, 17) identifizieren die Herausgeberinnen einige wenige einschlägige Studien aus der Zeit vor 2010, aber ansonsten thematisieren sie den Befund einer großen Leerstelle in diesem Themenfeld (vgl. auch Andresen/Künstler 2015). Das hat sich im Zuge der Forschungsförderlinien des BMBF zur sexualisierten Gewalt in pädagogischen Kontexten und nicht zuletzt durch verschiedene wissenschaftliche Studien zur Aufarbeitung etwa in kirchlichen Einrichtungen geändert. Seit dem Jahr 2010 ist folglich ein Wandel zu beobachten, der anhand von Forschungsprojekten, wissenschaftlichen Tagungen, Diskussionen, Publikationen und fachpolitischen Aktivitäten sichtbar wird. Diese Dynamik resultierte nicht primär aus einem genuin erziehungswissenschaftlichen Erkenntnisinteresse. Vielmehr wurde sie vor allem durch die Berichte von Menschen, die von Gewalt betroffen waren, engagierte journalistische Berichterstattung und Aufklärung, mediale Aufmerksamkeit und politischen Handlungsdruck in Gang gesetzt.

Der Blick auf Pädagogik als Gefahrenzone für Kinder und Jugendliche und die Bemühungen um die Stärkung pädagogischer Einrichtungen als Schutzund Bildungsräume ist auch mit der Analyse der Anerkennung und Gewährleistung von Rechten zu verbinden. Seit dem Jahr 2000 haben Kinder in Deutschland das Recht auf ein gewaltfreies Aufwachsen, auf Erziehung ohne Gewalt. Diese Ausrichtung gilt oft auch als Maßstab für die Aufarbeitung von Unrecht in der Kindheit (Andresen 2018a). Damit sind allerdings normative Setzungen verbunden, wie internationale Diskussionen im Rahmen von „Transitional Justice“ zeigen (Andresen 202ob). So problematisiert die norwegische Kriminologin Kjersti Ericsson (2015) den für Aufarbeitung in der Gegenwart liegenden Maßstab der Bewertung des Umgangs mit Kindern in 
(sozial)pädagogischen Einrichtungen: „By what normative yardstick should the near past be measured?" (ebd., 43)

$\mathrm{Zu}$ solchen systematischen und normativen Anfragen hätte die erziehungswissenschaftliche Forschung einiges beizutragen. Ein interdisziplinär angelegter Ansatz wird beispielsweise derzeit in dem BMBF-Verbundprojekt „Auf-Wirkung. Aufarbeitung für wirksame Schutzkonzepte in Gegenwart und Zukunft" erprobt. ${ }^{5}$ Ausgangspunkt für die auch erziehungswissenschaftlich orientierten Analysen im Rahmen des Projektes sind Berichte Betroffener und Erfahrungen mit internationaler Aufarbeitung und Erinnerungskultur. Das Projekt prüft empirisch, ob und wie aus Fehlern in der Vergangenheit für die Gestaltung von pädagogischen Einrichtungen und für das Generationenverhältnis gelernt werden kann.

In dem Frankfurter Teilprojekt der Autorinnen geht es um Erkenntnisse über soziale, kulturelle oder persönliche Bedingungen, die dazu beigetragen haben, dass sich Betroffene anderen anvertrauen. Für dieses Anvertrauen wird in der Forschungsliteratur der Fachbegriff „Disclosure“ verwendet. Er beschreibt den „Prozess des Offenlegens und der Hilfesuche durch eine betroffene Person bei realen Erfahrungen sexuellen Missbrauchs“, wobei die Offenlegung häufig „im Rahmen von Vertrauens-, Freundschafts- und Therapiebeziehungen“ erfolgt (Kindler/Schmidt-Ndasi 2011, 30).

In sozialwissenschaftlichen Abhandlungen, die sich mit dem Verhältnis zwischen Sprache und sexualisierten Gewalterfahrungen aus Sicht von Betroffenen auseinandersetzen, wird dies unter den unterschiedlichen Begriffen wie „Aufdeckung“, „Offenbarung“, „Offenlegung“" oder „Disclosure“ verhandelt. In dem Frankfurter Projekt wurde unter Rückgriff auf Mosser (2009) Disclosure als Prozess verstanden. Mosser identifiziert drei für das Anvertrauen zentrale Systeme bzw. "Systemkonfigurationen“ (ebd., 283). Relevant sei das "Missbrauchssystem“, das „Aufdeckungssystem“ sowie das „Hilfesystem“. Vor allem die Übergänge zwischen diesen Systemen im Sinne von Veränderungen innerhalb einer sozialen, einer innerpsychischen sowie einer ethischen Dimension werden von Mosser weiterführend beschrieben (ebd.). Die soziale Dimension umfasst dabei Fragen der Zugehörigkeit und Einflussnahme; die innerpsychische Dimension kognitive (Bewusstsein, Erklärung), als auch emotionale Erlebensaspekte (Angst, Ambivalenz). Die ethische Dimension bezieht sich auf Bewertungsprozesse, d.h. unter anderem auf Fragen der Schuld.

5 Siehe Fußnote 1. 
Sich einer oder mehreren Personen anzuvertrauen, etwa in einer Schule, ist meist kein genau abzugrenzendes Ereignis. Sprechen über sexualisierte Gewalt ist ein Prozess, der mit stetigen Entscheidungs- und Abwägungsüberlegungen einhergeht und mit unterschiedlichen Stationen der Biographie verbunden sein kann (Pohling 2021). Im Rahmen der Auswertungen von Anhörungen in diesem Projekt ist eine empirische Analyse von Vertrauen als eine von fünf Voraussetzungen für das Sprechen auch in pädagogischen Kontexten erfolgt (Gudat u.a. 2021). Dabei wurden drei Aspekte von Vertrauen herausgearbeitet, die das Sprechen über Gewalt eher ermöglichen: Es braucht Vertrauen in andere Menschen, in den Prozess sowie in die eigenen Kräfte (ebd.).

An diese Erkenntnisse aus der Auswertung von vertraulichen Anhörungen kann die Diskussion anschließen, in der es um Aufarbeitung der „Gefahrenzone“ Pädagogik sowie um die Gestaltung von Pädagogik als Schutzraum geht. Dabei können die drei rekonstruierten Aspekte von Vertrauen auf die Pädagogik übertragen werden und als Eckpunkte für die institutionelle Rahmung und Organisation von Kindertagesstätten, Schulen oder auch Heimeinrichtungen auch in kirchlicher Trägerschaft Geltung erlangen.

\section{Charakteristiken gesellschaftlicher Aufarbeitung sexuellen Kindesmissbrauchs in Deutschland}

Ein Kennzeichen des Umgangs mit sexueller Gewalt gegen Kinder und Jugendliche ist das Schweigen der anderen im Umfeld Betroffener und die Verschwiegenheit über dieses Unrecht auf gesellschaftlicher Ebene. ${ }^{6}$ Gleichwohl berichten Betroffene und andere Zeitzeug:innen davon, dass das Schweigen nicht unbedingt aus einem Mangel an Wissen resultierte. Das Verschweigen von Gewalt kann dort aufrecht erhalten werden, wo sich Täter:innen auf Schweigen, Vertuschen und Schutz verlassen und auf Netzwerke vertrauen können. Die Moralphilosophin Baier bezeichnet dies als Vertrauen „ohne Gerechtigkeit“:

„Die wenigen Diskussionen zum Vertrauen, die ich in der moralphilosophischen Literatur gefunden habe, gehen davon aus, dass Vertrauen ein Gut ist und dass die Enttäuschung eines bewussten Vertrauens prima facie immer schlecht, während die Erfüllung des Vertrauens prima facie immer richtig ist. Was aber ist eine durch Vertrauen gebundene Gemeinschaft ohne Gerechtigkeit, wenn nicht eine Gruppe von gegenseitigen Erpressern und Ausbeutern?

6 Die Gewaltforscher Eddie Hartmann und Thomas Hoebel (2020) bezeichnen dies als "Schweigsamkeit der Gewalt“ oder „verschwiegene Gewalt“. 
Wenn eine Vertrauensbeziehung in sich korrupt ist und die Brutalität, Tyrannei und Ungerechtigkeit fortsetzt, dann kann das Vertrauen eine alberne Selbstgefährdung sein und das Enttäuschen oder Verraten des Vertrauens [...] wird dann nicht nur moralisch erlaubt, es wird sogar moralisch lobenswert sein.“ (Baier 2001, 72)

Vertrauensbeziehungen, so Baier, sind folglich nicht per se moralisch intakt und es kann mehr als geboten sein, das Schweigen darüber zu brechen. Damit ist das Themenfeld der gesellschaftlichen Aufarbeitung angesprochen, auf das in diesem Abschnitt in gebotener Kürze am Beispiel der Unabhängigen Kommission zur Aufarbeitung sexuellen Kindesmissbrauchs eingegangen werden soll. Die Einrichtung der Kommission im Jahr 2016 schließt an international vergleichbare Vorgehensweisen an, Gewalt gegen Kinder und Jugendliche in pädagogischen Einrichtungen durch Erwachsene und andere Jugendliche sichtbar und öffentlich thematisierbar zu machen (Wright 2017). Die Unabhängige Kommission hat den Auftrag, Strukturen, die sexuellen Kindesmissbrauch ermöglicht und Vertuschung sowie unterlassene Hilfe nicht sanktioniert haben, in allen Bereichen der Gesellschaft aufzuarbeiten. ${ }^{7}$ Sie befasst sich mit der Familie als Tatkontext und untersucht Institutionen wie Schulen in staatlicher oder kirchlicher Trägerschaft, Heimeinrichtungen und auch Sportvereine (Unabhängige Kommission 2019a). ${ }^{8}$

Die Kommission führt seit 2016 vertrauliche Anhörungen mit betroffenen Menschen durch und nimmt schriftliche Berichte entgegen. Seitdem haben sich weit mehr als 2000 Betroffene, aber auch andere Zeitzeug:innen, etwa Lehrerinnen oder Familienangehörige betroffener Menschen, an die Kommission gewandt. In diesen ausführlichen Gesprächen und Berichten erfährt die Kommission stellvertretend für die Gesellschaft von erlittener sexueller Gewalt in der Kindheit, von unterlassener Hilfe im Umfeld, von Schuld- und Ohnmachtsgefühlen, aber auch von vielfältigen Versuchen, die Folgen zu bewältigen. Die Aufarbeitungskommissionen anderer Länder haben sich bisher primär mit sexuellem Kindesmissbrauch im institutionellen Bereich befasst (Wright 2017). Doch ein großer Anteil betroffener Menschen hat sexuellen Missbrauch in der Familie erlebt. Die Kommission in Deutschland untersucht daher auch den sexuellen Kindesmissbrauch innerhalb der

7 Siehe Kurzvorstellung „Unabhängige Kommission zur Aufarbeitung sexuellen Kindesmissbrauchs", einzusehen unter https://www.aufarbeitungskommission.de, zuletzt aufgerufen am 7.2.2021.

8 Die Mitglieder der Kommission kommen aus verschiedenen Arbeitsgebieten und sind ehrenamtlich tätig. Sie werden hauptamtlich unterstützt von einem Büro, begleitet von Betroffenen und dem Unabhängigen Beauftragten für Fragen des sexuellen Kindesmissbrauchs (UBSKM). 
Familie. Sie kann dabei zwar an frühe Studien aus der Frauen- und Selbsthilfebewegung anschließen (Kavemann/Lohstöter 1984), hat aber institutionell und konzeptionell Neuland betreten. ${ }^{9}$ Bearbeitet wurden u.a. sexueller Kindesmissbrauch in der DDR (Sachse/Knorr/Baumgart 2018; Unabhängige Kommission 2019c), in der katholischen und evangelischen Kirche in Deutschland und deren Umgang mit Tätern und Täterinnen und Betroffenen (Unabhängige Kommission 2019c) sowie organisierte sexuelle Gewalt (Nick u.a. 2018; 2019). Weitere Aufarbeitungs- und Forschungsschwerpunkte sind u.a. Missbrauch im Sport, Aufarbeitung in Schulen, Menschen mit Behinderung und sexuelle Gewalt. Im Anschluss an das dritte öffentliche Hearing der Aufarbeitungskommission zu „Kirchen und ihre Verantwortung für Aufarbeitung sexuellen Kindesmissbrauchs" im Juni 2018 hat der Unabhängige Beauftragte für Fragen sexuellen Kindesmissbrauchs eine „Arbeitsgruppe Kirchen“ unter Beteiligung von drei Kommissionsmitgliedern eingerichtet. ${ }^{10}$ Ein Ergebnis ist die so genannte „Gemeinsame Erklärung über verbindliche Kriterien und Standards für eine unabhängige Aufarbeitung von sexuellem Kindesmissbrauch in der katholischen Kirche in Deutschland" zwischen dem UBSKM und der Deutschen Bischofskonferenz."

\section{Schriftliche Berichte an die Aufarbeitungskommission und methodisches Vorgehen bei ihrer Auswertung}

Neben vertraulichen Anhörungen können sich Betroffene und andere Zeitzeug:innen auch schriftlich an die Kommission wenden und ihre Geschichte hinterlegen. Diese Dokumente haben einen anderen Charakter

9 Siehe auch das Impulspapier des Betroffenenrats beim UBSKM vom 15.3.2021, einzusehen unter https://beauftragter-missbrauch.de/betroffenenrat/der-betroffenenrat/ aus-unserer-sicht/detail?tx_news_pi1 ${ }_{0} 5$ Baction $\%{ }_{5} \mathrm{D}=$ detail\&tx_news_pi1\% ${ }_{5} \mathrm{~B}$ controller\% $\% 5 \mathrm{D}=$ News\&tx_news_pi1 $\% 5$ Bnews $\%{ }_{5} \mathrm{D}=492 \& \mathrm{cHash}=$ fig2dd $56 \mathrm{bd} 53 \mathrm{c} 251 \mathrm{c} 4 \mathrm{e} 7 \mathrm{O}$ 2e24eb58o68, zuletzt aufgerufen am 2.4.2021.

10 Siehe „Kirchen und ihre Verantwortung zur Aufarbeitung sexuellen Kindesmissbrauchs: 3. Öffentliches Hearing der Aufarbeitungskommission in Berlin“, 27.6.2018, einzusehen unter https://www.aufarbeitungskommission.de/service-presse/service/meldungen/ oeffentliches-hearing-kirchen, zuletzt aufgerufen am 2.4.2021.

11 Siehe „Gemeinsame Erklärung über verbindliche Kriterien und Standards für eine unabhängige Aufarbeitung von sexuellem Missbrauch in der katholischen Kirche in Deutschland des Unabhängigen Beauftragten für Fragen des sexuellen Kindesmissbrauchs und der Deutschen Bischofskonferenz", 28.4.2020, einzusehen unter https:// www.dbk.de/fileadmin/redaktion/diverse_downloads/presse_2020/2020-o74a-Gemeins ame-Erklaerung-UBSKM-Dt.-Bischofskonferenz.pdf, zuletzt aufgerufen am 2.4.2021. 
als die Transkripte der ausführlichen Gespräche oder Dokumentationen der öffentlichen Hearings. Eine erste Charakterisierung wurde im Bilanzbericht (Unabhängige Kommission 2019a und b) vorgenommen. Zudem wurde eine Auswertung zur Beschreibung des Kindes, das man einst war, in den schriftlichen Dokumenten vorgelegt (Andresen 2018b). Anfang 2021 lagen der Kommission knapp 470 schriftliche Berichte vor. Auf ihrer Internetseite werden Orientierungsfragen angeboten, und zwar unterschieden nach Gewalterfahrungen in der Familie und in anderen Institutionen. ${ }^{12} \mathrm{Zu}$ den Themen der Leitfragen gehören Tatgeschehen („was geschah“), Anvertrauen/ Disclosure, Reaktionen der Institution bzw. Familie und soziales Umfeld, Einschaltung von Behörden, Tatfolgen, Botschaften an Politik, Gesellschaft und an die Kommission. Diese versucht über ihre Kommunikationskanäle, etwa die Internetseite, Haltung, Vorgehensweise und Anerkennung deutlich zu machen und um Vertrauen zu werben:

"Sie haben sich entschieden der Kommission über sexuellen Missbrauch zu berichten und möchten dies schriftlich tun. Das ist ein mutiger und wichtiger Schritt. Ihr Bericht wird der Kommission helfen herauszufinden, was sexuelle Gewalt in der Kindheit ermöglicht hat und was Politik und Gesellschaft verändern müssen, damit Kinder in Zukunft besser vor Missbrauch geschützt sind. Bitte schreiben Sie uns in Ihren eigenen Worten. Dabei macht es nichts, wenn Sie sich nicht an alles erinnern können. Um Sie beim Schreiben zu unterstützen, finden Sie ab Seite 2 einige Fragen, an denen Sie sich in Ihrem Bericht orientieren können, aber natürlich nicht müssen. Manche Fragen sind für Sie vielleicht weniger wichtig oder gar nicht zutreffend. Sie allein entscheiden, was Sie der Kommission mitteilen möchten. Sie können den Bericht mit Ihren Kontaktdaten versehen oder anonym bleiben. Wenn Sie uns mitteilen, wie Sie erreichbar sind, hat die Kommission die Möglichkeit Sie zu kontaktieren, falls es Rückfragen gibt. ${ }^{\text {13 }}$

Mehr als 470 vorliegende schriftliche Berichte sind in einem Aufarbeitungsprozess ein starkes Signal, mit dem die jeweiligen Autor:innen stellvertretend für die Gesellschaft die Kommission in die Verantwortung nehmen und ihr Vertrauen entgegenbringen. Die Betroffenen charakterisieren das Schreiben selbst als einen zuweilen längeren Prozess, in dem sie auch in einen inneren Dialog mit der Kommission treten. Für einige ist diese schriftliche Kommunikation mit einem um Vertrauen werbenden Gremium teils langwierig, auch schmerzhaft und mühsam, andere erleben das Schreiben als befreiend. Es gibt, anders

12 Siehe „Schriftlicher Bericht - Erfahrungen mit sexuellem Kindesmissbrauch aufschreiben“, einzusehen unterhttps://www.aufarbeitungskommission.de/ihre-geschichte/ schriftlicher-bericht, zuletzt aufgerufen am 2.4.2021.

Ebd. 
als bei den vertraulichen Anhörungen, kein direktes Gegenüber und damit auch keinen unmittelbar erlebbaren Resonanzraum. Gleichwohl wird die Kommission in den Berichten direkt angesprochen und auch aufgefordert, sich weiter im Kampf gegen Missbrauch einzusetzen und die Bereitschaft von Betroffenen, ihre Geschichten mitzuteilen, als Vertrauensvorschuss zu bewerten. Mit der oben eingeführten Denkfigur, dass Vertrauen geschenkt wird und den Beschenkten eine besondere Verantwortung zukomme, wird gerade in den schriftlichen Berichten die Dimension Verantwortung besonders sichtbar, ebenso wie die Verschränkung von Privatem und Öffentlichen. ${ }^{14}$

Viele Betroffene orientieren sich nicht an den Leitfragen, sondern folgen als Autor:innen ihren eigenen Erzählsträngen. Dadurch stellen diese Dokumente für die wissenschaftliche Auswertung eine Herausforderung dar. Für diesen Beitrag wurden 102 schriftliche Berichte von Menschen im Alter zwischen 25 und 9o Jahren herangezogen. Die Gewalterfahrungen, die Dauer und Intensität sowie die Tatkontexte weisen große Unterschiede auf. Es sind auch hier viele Betroffene vertreten, die sexuelle Gewalt im familiären Umfeld erlebt haben, aber es wird auch über sexuelle Gewalterfahrungen in anderen Tatkontexten wie Schulen oder Vereinen berichtet.

Ein Auswertungsfokus lag auf Vertrauen, weil dies eine wichtige Kategorie für Disclosure-Prozesse darstellt. Die Berichte wurden zunächst daraufhin ausgewertet, ob in ihnen Begriffe aus der Wortfamilie Vertrauen auftauchen, etwa Misstrauen, anvertrauen, vertraut, vertraulich etc. Dies war in 36 Berichten der Fall. Aus diesen sind diejenigen Berichte vertieft in die Analyse, die für diesen Artikel vorgenommen wurde, einbezogen worden, in denen Vertrauen als Schlüsselthema des gesamten Berichts identifiziert werden konnte. Dies war in zehn Berichten der Fall. Acht Frauen und zwei Männer, geboren zwischen 1946 und 1990, haben die Berichte verfasst. ${ }^{15}$ Vier Betroffene berichten von mehr als einem Täter, in einem Bericht geht es ausführlich um die Mittäterschaft der leiblichen Mutter. In fünf Berichten wird vor allem über die Familie als Tatkontext berichtet, eine Person berichtet über einen Kindergarten als Tatkontext, eine weitere über ein katholisches Internat, zwei Betroffene berichten von medizinischen Kontexten bzw. Situationen, und eine vom Musikunterricht. In den meisten Berichten wird auch über die Schule und Reaktionen von Lehrkräften gesprochen. Im letzten Auswertungsschritt der

14 Zu Übergängen zwischen privater bzw. persönlicher Aufarbeitung und öffentlicher Aufarbeitung arbeitet im Teilprojekt 5 besonders Bastian Schwennigcke (Universität Rostock) in seiner Dissertation.

15 In der Pseudonymisierung wird eine Jahresspanne von fünf Jahren angegeben z.B. geboren zwischen 1946 und 1950 . 
Verdichtung sind wieder alle Berichte hinzugezogen worden. In dem deduktivinduktiven Vorgehen bei der Auswertung der schriftlichen Berichte sind unterschiedliche Verortungen des Schreibens über Vertrauen sichtbar geworden. Die in der systematischen Literatur zu Vertrauen auffindbare Unterscheidung zwischen Vertrauen bezogen auf andere Menschen, auf sich selbst und auf Prozesse und Institutionen lassen sich auch anhand der Feinanalyse von Schlüsselzitaten aus 36 schriftlichen Berichten aufzeigen.

\section{Narrative von Vertrauen in Erziehungsverhältnissen und sexuelle Gewalt}

Betroffene werden in und mit ihren Berichten an die Aufarbeitungskommission zu Zeug:innen von Vertrauen, Verletzbarkeit und Verantwortung. Deren Thematisierung ermöglicht, das Schmerzhafte und Verletzliche sichtbar und sagbar zu machen. In der verdichteten Darstellung der Ergebnisse sollen drei Narrative hervorgehoben werden: Erstens das Narrativ des missbrauchten Vertrauens des betroffenen Kindes, in dem auch eine normative Aufladung von Kindheit und die Beschreibung des Kindes aufscheint, das man einst war (Andresen 2018b). Zweitens das Narrativ des erschlichenen Vertrauens als Strategie von Täter:innen und drittens das Narrativ des Vertrauensklimas, in dem Sprechen und Anvertrauen im sozialen Umfeld ermöglicht oder behindert wurde. Alle drei Narrative sind für pädagogische Kontexte und Einrichtungen relevant, auch bei Fällen sexueller Gewalt in der Familie. So zeigt eine jüngst publizierte Auswertung von Anhörungen und schriftlichen Berichten, dass nahezu alle Betroffenen ihre Schulzeit thematisieren. Sie sprechen über Schule als Tatort, als Schutzraum und als Lern- und Bildungsort (Andresen/ Bauch 2021, 5).

\section{VI.1 Missbrauchtes Vertrauen}

Um zu beschreiben, wie tief die Gewalterfahrung ihre gesamte Persönlichkeit getroffen und verletzt hat, nutzen Betroffene den Begriff des „missbrauchten Vertrauens". Wie zu Beginn des Beitrags hergeleitet, sind Kinder existenziell darauf angewiesen, Erwachsenen in ihrer unmittelbaren Umgebung zu vertrauen. Vertrauen charakterisiert einen wesentlichen Teil von Erziehungsverhältnissen, denn es sind die anderen, die einem Kind etwas zeigen, ihm etwas erklären, ihm Normen und Werte vermitteln. Insbesondere jüngere Kinder, denen es an Überblick und Lebenserfahrung fehlt, werden häufig als "naiv" beschrieben, und zwar aufgrund ihres fehlenden Wissens und ihres „treuen“ Glaubens an Ältere. Betroffene führen so die Tragweite missbrauchten 
Vertrauens für ein Kind vor Augen, denn Grenzverletzungen und sexuelle Gewalt gegen Kinder erschüttern das Verhältnis von Ich und Umwelt. In den schriftlichen Berichten, aber auch in den Anhörungen beschreiben Erwachsene eindrücklich, wie sich durch sexuelle Gewalt ihr Blick auf die Familie, die Gleichaltrigen, ihren Alltag veränderte. Kinder sind zunächst bereit, Erwachsenen Wohlwollen zu unterstellen. Zu erfahren und zu wissen, dass ein Vertrauensverhältnis ausgenutzt und der Spielraum und das Machtgefälle für Grenzüberschreitungen und sexuelle Gewalthandlungen genutzt werden, hat aus der Perspektive eines Kindes und seiner spezifischen Verletzbarkeit eine existenzielle Seite.

Doch Vertrauensmissbrauch können auch Mütter und Väter oder andere, einem Kind nahestehende Personen empfinden, die im Vertrauen auf das Wohlwollen einem Täter das Kind leichtgläubig anvertraut haben. Baier (2001) erklärt aus ihrer moralphilosophischen Systematik heraus dieses Phänomen damit, dass auch Eltern in ihrem Verhältnis zum Kind ein leicht zerbrechliches Vertrauen haben. Für betroffene Kinder ist diese Leichtgläubigkeit der Erwachsenen häufig einschüchternd und für Erwachsene führt sie zum Ausblenden des Möglichen. Dies berichtet eine Betroffene, geboren zwischen 1966 und $1970^{16}$ über ihre Familie:

„Ich denke, das Vertrauen, dass der Heilpraktiker bei meiner Großtante und meinen Eltern genoss. So hat meine Tante mich vermutlich ohne Bedenken mit ihm allein gelassen. Meine Eltern vertrauten ihm auch. So konnte ich mir vermutlich auch nicht vorstellen, dass er etwas Verbotenes tut." (Betroffene)

Diese Betroffene thematisiert, wie stark Netzwerke von Erwachsenen den weiteren Verlauf einer Gewaltgeschichte beeinflussen. Hier ist das Vertrauen als eine Ressource für den Täter und eine Hürde für das Kind zu verstehen, das mit ermöglicht, dass der Heilpraktiker "etwas Verbotenes tut“. Es wird zudem deutlich, wie stark soziale Statusfragen und die Anerkennung von Autorität etwa eines Priesters einen Vertrauensschutz für Täter:innen darstellen. Wenn das nahe Umfeld Täter:innen großes Vertrauen entgegenbringt, ist die Position eines Kindes außerordentlich geschwächt (s.a. Andresen 2018b). Dies führt vielfach dazu, dass selbst in einem Moment des Anvertrauens dem Kind gegenüber Zweifel und Unglaube artikuliert werden. Die oft erst späte Erkenntnis über missbrauchtes Vertrauen geht auf Seiten der für Kinder Verantwortlichen häufig mit Schuldgefühlen einher.

16 Aus daten- und personenschutzrechtlichen Gründen werden Geburtsjahrgänge mit der Zeitspanne von fünf Jahren angegeben. 


\section{VI.2 Erschlichenes Vertrauen}

Betroffene verfügen über sehr viel Wissen, wenn es um die Strategien von Täter:innen geht.

„Er war Angestellter am Kindergarten (Hausmeister), kein Kindergärtner, doch es bestand eine gewisse emotionale Verbindung. Er war mir also nicht fremd, sondern vertraut, jemand, der zum bekannten Umfeld gehörte." (Betroffene)

Sich um das Vertrauen nicht nur naher Familienangehöriger, sondern von Kindern und Jugendlichen selbst zu bemühen, gehört zu den häufig berichteten Strategien von Täter:innen. Im Narrativ des erschlichenen Vertrauens werden die Komplexität von Vertrauen und das Risiko der Verletzbarkeit sichtbar, insbesondere bei ungleichen Machtverhältnissen. Die oben zitierte Betroffene stellt hier Vertrautheit und Fremdheit einander gegenüber. Das verweist auch auf ein gängiges gesellschaftliches Narrativ, denn die Vorstellung, dass der „fremde Täter" Kinder und Jugendliche bedrohe, ist nach wie vor vorhanden.

Insbesondere in persönlichen oder familiären Krisensituationen ist es für Kinder und Jugendliche wichtig, Menschen zu haben, auf die sie sich verlassen und denen sie vertrauen können. Hier liegt eine der perfiden Möglichkeiten von Täter:innen, sich ihnen zu nähern. Dies ist eine Strategie, die insbesondere in pädagogischen Einrichtungen oder von Verwandten außerhalb der Kernfamilie genutzt werden kann. Dafür liegen sehr viele Beispiele aus Anhörungen und Berichten vor. Ein Betroffener, der in einem katholischen Internat von einem Erzieher sexuelle Gewalt erlebt hat, beschreibt, wie abhängig er davon war, dass sich ihm jemand mit Interesse zuwendet:

„Der geregelte Tagesablauf, Erfolgserlebnisse in der Schule, Akzeptanz in der Gemeinschaft haben mich aus einer sehr schwierigen persönlichen Situation heraus wieder auf die Bahn gebracht. Eine wichtige Rolle spielte dabei *(Täter), der mit viel Einsatz und Verständnis für mich schnell zu einer unzweifelhaften Vertrauensperson wurde.“

In diesem Ausschnitt zeigt sich zudem, in welcher Form geregelte Strukturen in einer Schule ein Klima des Vertrauens schaffen können für Kinder und Jugendliche, denen aus unterschiedlichen Gründen eine äußere und/oder innere Strukturiertheit fehlte. Dies trug aus der Sicht des Betroffenen in diesem Fall dazu bei, dass der Täter sogar zu einer „unzweifelhaften Vertrauensperson“ werden und der im Zuge der verschiedenen Tatgeschehen von dem Jugendlichen auch "Vertrauensbeweise“ einfordern konnte, ohne befürchten zu müssen, dass es zu einer Aufdeckung kommt. Sich für Kinder und Jugendliche vermeintlich individuell zu interessieren und so Vertrauen aufzubauen, gehört 
zu den weiteren Einfallstoren, wie der Bericht einer Betroffenen zeigt, deren Täter in einer kirchlichen Gemeinde Musiker war:

„Herr *(Täter) war auch einer der wenigen Erwachsenen in dieser Zeit, der sich für die Probleme der Kinder und Jugendlichen interessierte. Ich vertraute ihm meine Sorgen an."

\title{
VI.3 Vertrauensklima
}

In dem Verhältnis von Vertrauen und Verantwortung geht es um die Gestaltung von Beziehungen, um Vorgehensweisen, um für Kinder transparente Strukturen und für sie zugängliche Informationen. Dies wird hier mit dem Begriff des Vertrauensklimas erfasst. Eine Betroffene, die in ihrer Familie sexuelle Gewalt erlitten hat, berichtet von einem widersprüchlichen Klima in ihrer Schule:

\begin{abstract}
„Meine LehrerInnen in der Grundschule haben mich gut behandelt. Das waren zwar alles alte Nazileute, wir haben z.B. das Deutschlandlied mit allen drei Strophen gelernt und sowas, aber mich als ,armes Kommunistenkind' haben die irgendwie unter ihre Fittiche genommen - das war im konservativen *(Stadt) nicht selbstverständlich. Mitschüler bekamen den vollen Rassismus des Klassenlehrers ab oder den geworfenen Schlüssel oder den Zeigestock neben die Finger. Meine Musiklehrerin hat mir nach der Schule Flöte beigebracht, und ich glaube, dass mich die Musik gerettet hat. Ich war eine ruhige, fleißige, sehr gute Schülerin. Schule hat mir Halt gegeben, dort spürte ich mich, ich war leistungsstark und interessiert. Aber ich wäre nie auf die Idee gekommen, mich dort anzuvertrauen. Ich hatte nirgendwo die Idee, dort könnte ich was sagen, nur bei meiner Mutter, der eine Versuch."
\end{abstract}

Im Zuge von Gewalterleben und Vertrauensmissbrauch ist es für Betroffene vor allem zentral, ob sie Menschen in ihrem Umfeld die Gewalterfahrungen anvertrauen können, damit sie aufhören. Das Zitat der Betroffenen verdeutlicht die Ambivalenz in den Berichten über das Vertrauensklima, denn einerseits geben ihr Strukturen und Personen in der Schule einen vertrauensvollen Rahmen, innerhalb dessen sie stärkende Erfahrungen sammeln kann. Andererseits aber ist es für das Mädchen undenkbar, sich dort anzuvertrauen und Hilfe zu suchen. Sie habe nicht die Idee gehabt, dort etwas sagen zu können und es liegt die Vermutung nahe, dass sie in diesem Umfeld lediglich selektiv vertrauen konnte und auch negative Erfahrungen gesammelt hatte.

Ein für Betroffene positives Vertrauensklima kann auch eine Bedeutung für das Erinnern und Versprachlichen haben (Lorenz 2020; Pohling 2021). Hierzu berichten Betroffene auch von bestimmten Schlüsselereignissen, über die die langjährige Macht von Tätern auch noch über erwachsene Menschen erkennbar wird. Die Betroffene, die im Kindergarten sexuelle Gewalt erlitten hat, schreibt dazu Folgendes: 
„Als wenn es tatsächlich all die Jahre über eine Art Verbindung gegeben hätte, etwas, das mich blockiert hat zu erinnern und zu erzählen, und das sich erst mit dem Tod des Mannes gelöst hat. Ich erwähne das deshalb, weil es - auch im Hinblick auf die gefährdeten Kinder von heute - so schrecklich ist zu erkennen, wie verwoben die Verhältnisse sind, die Missbrauch ermöglichen, und wie schwer es für die „Opfer“ sein kann, sich anzuvertrauen oder überhaupt Worte für das zu finden, was passiert ist oder droht zu passieren."

In dem ausführlichen Bericht einer Frau, die in einem religiösen familiären Milieu jahrelang sexueller Gewalt ausgeliefert war, gibt die Betroffene Hinweise darauf, wie sehr Kinder darauf angewiesen sein können, zu jemandem außerhalb der Familie Vertrauen fassen zu können.

\begin{abstract}
„Meine Lehrer hatten damals gemerkt, dass ich Probleme habe, sie hatten meine Mutter gefragt warum ich so still bin. Da ich meinen Eltern nicht vertrauen konnte hätte es mir geholfen Vertrauen zu einem Experten aufzubauen, das hätte mich vor vielem Schaden bewahren können. In meinem Fall war die Familiendynamik und Kontrolle sehr machtvoll. Meine Eltern hätten mich nie freiwillig zu einem Psychologen geschickt."
\end{abstract}

Doch es finden sich auch viele Schilderungen darüber, dass dem Umfeld nicht vertraut werden konnte und Versuche des Anvertrauens gerade auch in der Schule abgewiesen wurden. Für betroffene Kinder und Jugendliche wirkte dies wie ein erneuter Vertrauensmissbrauch, wie ein schwer zu durchbrechender Teufelskreis. Eine Betroffene, die als Kind eine körperliche Behinderung hatte und von mehreren Tätern sexuelle Gewalt erlitten hat, berichtet ausführlich über ihre negativen Erlebnisse mit der Mutter und über deren Rolle als Unterstützerin im Tatgeschehen. In diesem Bericht werden die Umstände deutlich beschrieben, durch die das Kind Schritt für Schritt jedes Vertrauen verliert:

„Sollte ich es doch noch einem sagen, noch einmal das Risiko einer furchtbaren Katastrophe eingehen und mich - ein unerhörter Gedanke! - einem Außenstehenden anvertrauen? Vielleicht geschah ein Wunder. Wenn nicht, hatte ich wirklich nichts unversucht gelassen. Dann musste, dann konnte und würde ich in Frieden den letzten Schritt tun.

Dann kam die Lehrerin. Sie gab mir ein Taschentuch und wartete, bis ich mir die Nase geputzt hatte. Sie ließ mich ausreden, und ich sagte ihr, was ich damals meiner Mutter gesagt hatte: ,Mein Stiefvater macht das mit mir, was man macht, wenn man Kinder haben will.' Es ging leichter, als ich gedacht hatte, ich hatte es ja auch schon geübt. Sie nickte und redete ein bisschen und sagte dann, ganz nebenbei und recht freundlich, dass sie sich ,nicht in Privatangelegenheiten einmischen' dürfe. Ich nickte und konnte den dicken Kloß im Hals nicht runterschlucken. Noch heute liegt mir das Wort Privatangelegenheiten auf der Seele. Ich weiß noch meinen ersten Gedanken dazu: Siehst du, das darf der tun, es ist in Wirklichkeit gar kein Verbrechen. Du denkst nur, dass es eins ist.“ 
Mit diesem Zitat einer Frau, die zwischen 1956 und $196 \mathrm{o}^{17}$ geboren wurde, kann an die oben geführte Diskussion zu „Pädagogik als Gefahrenzone“ angeschlossen werden. Sichtbar wird eine vermutlich historisch weit in die Geschichte der bürgerlichen Gesellschaft zurückreichende Vorstellung von der Privatheit sexueller Gewalt, die so zu einer "verschwiegenen Gewalt“ (Hartmann/Hoebel 2020) wird.

\section{Vertrauen, Verletzbarkeit und gesellschaftliche Aufarbeitung - Resümee}

In diesem Beitrag wurde versucht, über das Verhältnis von Vertrauen und Verletzbarkeit „Pädagogik als Gefahrenzone“ auszuleuchten. Dabei ging es um die Angewiesenheit des Kindes, vertrauen zu können und die Fähigkeit und den Willen Erwachsener, diesem Vertrauen so zu begegnen, dass keine erneuten Verletzungen entstehen. In den schriftlichen Berichten an die Kommission sprechen Betroffene über das zerstörte, missbrauchte Vertrauen des einstigen Kindes. Sie beschreiben, wie Familienangehörige und Pädagog:innen versagten, als ihnen Vertrauen entgegengebracht wurde, ein Kind sich ihnen anvertraut hat. Ein eindrucksvolles Beispiel dafür ist der Bericht über die Lehrerin, die zwar vordergründig verständnisvoll reagiert, sich aber weigert, in einer „Privatangelegenheit“ tätig zu werden. An dieser Geschichte zeigt sich, dass Familie und pädagogische Einrichtungen wie die Schule in Aufarbeitungsprozessen zusammen betrachtet werden müssen. Die in der Analyse herausgearbeiteten drei Narrative missbrauchtes Vertrauen, erschlichenes Vertrauen und Vertrauensklima verweisen auf Handlungsmöglichkeiten in pädagogischen Kontexten, um Kinder und Jugendliche hier und heute besser zu schützen. Wie Pädagogik die Erkenntnisse nutzen wird, muss man kritisch beobachten.

Das BMBF-Forschungsprojekt „Auf-Wirkung“ liegt an der Schnittstelle von gesellschaftlicher Aufarbeitung und Gewaltforschung. Aufarbeitung sexueller Gewalt findet bislang weitgehend unabhängig von der sozialwissenschaftlichen Gewaltforschung statt, bietet aber wichtige Impulse (Andresen 2020a). Ein solcher liegt möglicherweise im Nexus von Vertrauen, Verletzbarkeit und Verantwortung. Jan Philipp Reemtsma hatte 2008 das Verhältnis von „Vertrauen und Gewalt" thematisiert. Er definierte Vertrauen in Anlehnung

17 Aus daten- und personenschutzrechtlichen Gründen werden Geburtsjahrgänge mit der Zeitspanne von fünf Jahren angegeben. 
an Luhmann (2001) als stabile wechselseitige Erwartungen darauf, dass das Gewaltmonopol des Staates die Bürger:innen schützt. Doch Reemtsmas Analyse gibt keine Antworten auf die in der Gewaltforschung eher marginalisierte „verschwiegene Gewalt" (Hartmann/Hoebel 2020), also Gewalt in häuslicher Umgebung gegen Frauen, Kinder und besonders abhängige Menschen oder Gewalt gegen Schutzbefohlene in pädagogischen Einrichtungen. Für diese Gewaltkontexte bietet der Grundlagenartikel von Baier (2001) fundierte Anschlussmöglichkeiten, was in diesem Beitrag gezeigt werden sollte.

In dem Anliegen gesellschaftlicher Aufarbeitung sexueller Gewalt gegen Kinder und Jugendliche wird das Verhältnis von Vertrauen, Verletzbarkeit und Verantwortung sowohl als gesellschaftliche Dimension als auch in seiner Tragweite für persönliche und asymmetrische Beziehungen und den darauf fußenden Alltag sichtbar und thematisierbar. Aufarbeitungsprozesse selbst sind mit dieser Dynamik konfrontiert. Betroffene müssen den Akteur:innen der Aufarbeitung vertrauen können: sei es den heute Verantwortlichen in Institutionen, den Beteiligten in Aufarbeitungsteams, dem Beteiligungsprozess oder den rechtlichen Möglichkeiten.

\section{Literaturverzeichnis:}

Andresen, Sabine/Künstler, Sophie (2015): Vulnerabilität und sexuelle Gewalt in der Kindheit. Herausforderungen der Kindheitsforschung, in: Zeitschrift für Sexualforschung 28(4), 318-334.

Andresen, Sabine (2018a): Revisiting the Child from Back Then. Reports on Sexual Abuse in Childhood and Systematic Perspectives on Vulnerability, in: Childhood Vulnerability Journal 1(1), 51-66.

Andresen, Sabine (2018b): Gewalt in der Erziehung als Unrecht thematisieren. Perspektiven aus der Aufarbeitung sexuellen Kindesmissbrauchs, in: Zeitschrift für Pädagogik 64(1), 6-14.

Andresen, Sabine (2020a): Aufarbeitung sexueller Gewalt gegen Kinder. Potenziale für die sozialwissenschaftliche Gewaltforschung, in: Westend. Neue Zeitschrift für Sozialforschung (17)1, 103-113.

Andresen, Sabine (2020b): Zeugnis ablegen - Zeugnis aufbewahren. Gesellschaftliche Aufarbeitung sexueller Gewalt gegen Kinder und Jugendliche und die Rolle der Archive, in: dies./Johannes Kistenich-Zerfaß (Hrsg.): Archive und Aufarbeitung sexuellen Kindesmissbrauchs. Dokumentation einer Tagung der Unabhängigen Kommission zur Aufarbeitung sexuellen Kindesmissbrauchs und des Hessischen Staatsarchivs. Darmstadt, 191-210. 
Andresen, Sabine/Bauch, Ricarda (2021): Wenn die Schule kein Schutzraum ist. Betroffene berichten über sexuelle Gewalt in der Schule, in: Die Grundschulzeitschrift 326 (Ausgabe „Sexueller Missbrauch“), 4-8.

Baier, Annette (2001): Vertrauen und seine Grenzen, in: Martin Hartmann/Claus Offe (Hrsg.): Vertrauen. Die Grundlage des sozialen Zusammenhalts. Frankfurt a. M.: Campus, 37-84.

Bartmann, Sylke/Pfaff, Nicolle/Welter, Nicole (2012): Vertrauen in der erziehungswissenschaftlichen Forschung, in: Zeitschrift für Pädagogik 58(6), 772-783.

Brumlik, Micha (2017):Advokatorische Ethik. Zur Legitimation pädagogischer Eingriffe. Neuausgabe mit einem Vorwort zur dritten Auflage. Hamburg: CEP Europäische Verlagsanstalt.

Bütow, Birgit (2012): Sexuelle Gewalt in der Heimerziehung. Ein Versuch, die pädagogische Kategorie des Vertrauens in die Analyse einzuführen, in: Zeitschrift für Pädagogik 58(6), 824-836.

Erikson, Erik H. (2005): Kindheit und Gesellschaft. 14. Aufl. Stuttgart: Klett-Cotta.

Fabel-Lama, Melanie/Tiefel, Sandra/Zeller, Maren (2012): Vertrauen und Profession.

Eine erziehungswissenschaftliche Perspektive auf theoretische Ansätze und empirische Analysen, in: Zeitschrift für Pädagogik 58(6), 799-811.

Finkelhor, David (2008): Childhood Victimization: Violence, Crime, and Abuse in the Lives of Young People. New York: Oxford University Press.

Gudat, Rebecca/Schaumann, Nina/Nagel, Bianca/Schoon, Wiebke/Reimann, Daniel/ Schwennigcke, Bastian (2021): Über sexualisierte Gewalt sprechen. Was Kinder dafür brauchen, in: Die Grundschulzeitschrift 326 (Ausgabe „Sexueller Missbrauch“), 20-23.

Hartmann, Eddie/Hoebel, Thomas (2020): Einleitung. Gewalt - Grenzen der Erklärbarkeit?, in: WestEnd. Neue Zeitschrift für Sozialforschung 17(1), 67-69.

Herzog, Lisa (2013): Persönliches Vertrauen, Rechtsvertrauen, Systemvertrauen. Zur Natur von Kredit und den Ursachen der Finanzkrise, in: Deutsche Zeitschrift für Philosophie 61(4), 529-548.

Jud, Andreas (2014): Sexueller Kindesmissbrauch - Begriffe, Definitionen und Häufigkeiten, in: Jörg M. Fegert/Ulrike Hoffmann/Elisa König/Johanna Niehus/Hubert Liebhardt (Hrsg.): Sexueller Missbrauch von Kindern und Jugendlichen. Ein Handbuch zur Prävention und Intervention für Fachkräfte im medizinischen, psychotherapeutischen und pädagogischen Bereich. Heidelberg: Springer, 41-49.

Kavemann, Barbara/Lohstöter, Ingrid (1984): Väter als Täter. Sexuelle Gewalt gegen Mädchen. „Erinnerungen sind wie eine Zeitbombe“. Reinbek bei Hamburg: Rowohlt. Lorenz, Friederike (2020): Der Vollzug des Schweigens. Konzeptionell legitimierte Gewalt in den stationären Hilfen. Wiesbaden: Springer VS.

Mosser, Peter (2009): Wege aus dem Dunkelfeld. Aufdeckung und Hilfesuche bei sexuellem Missbrauch an Jungen. Dissertationsschrift. Wiesbaden: VS Verlag für Sozialwissenschaften. 
Nick, Susanne/Schröder,Johanna/Briken, Peer/Richter-Appelt, Hertha (2018): Organisierte und rituelle Gewalt in Deutschland. Kontexte der Gewalterfahrungen, psychische Folgen und Versorgungssituation, in: Trauma und Gewalt 12(3), 244-261.

Nick, Susanne/Schröder,Johanna/Briken, Peer/Richter-Appelt, Hertha (2019): Organisierte und rituelle Gewalt in Deutschland. Praxiserfahrungen, Belastungen und Bedarfe von psychosozialen Fachkräften, in: Trauma und Gewalt 13(1), 114-127.

Pohling, Andrea (2021): Artikulationen Sexueller Gewalt. Biographien, Diskurse und der Übergang zum Sprechen. Wiesbaden: Springer VS. Dissertationsschrift GoetheUniversität Frankfurt a. M. (im Erscheinen).

Reichenbach, Roland (2011): Pädagogische Autorität: Macht und Vertrauen in der Erziehung. Stuttgart: Kohlhammer.

Retkowski, Alexandra/Treibel, Angelika/Tuider, Elisabeth (2018): Handbuch sexualisierte Gewalt und pädagogische Kontexte. Theorie, Forschung und Praxis. Weinheim/ Basel: Beltz Juventa.

Sachse, Christian/Knorr, Stefanie/Baumgart, Benjamin (2018): Sexueller Missbrauch in der DDR. Historische, rechtliche und psychologische Hintergründe des Sexuellen Missbrauchs an Kindern und Jugendlichen in der DDR. Wiesbaden: Vieweg.

Spitz, René A. (1976): Vom Säugling zum Kleinkind. Naturgeschichte der Mutter-KindBeziehungen im ersten Lebensjahr. Unter Mitarbeit von W. Godfrey Cobliner. 5. Aufl. Stuttgart: Ernst Klett.

Unabhängige Kommission zur Aufarbeitung sexuellen Kindesmissbrauchs (2019a): Bilanzbericht. Bd 1, 20.12., einzusehen unter https://www.aufarbeitungskommission. de/wp-content/uploads/2019/o5/Bilanzbericht_2019_Band-I.pdf, zuletzt aufgerufen am 8.11.2020.

Unabhängige Kommission zur Aufarbeitung sexuellen Kindesmissbrauchs (2019b): Bilanzbericht. Bd. 2, 20.12. einzusehen unter https://www.aufarbeitungskommission.de/wp-content/uploads/2019/o5/Bilanzbericht_2019_Band-II.pdf, zuletzt aufgerufen am 8.11.202O.

Unabhängige Kommission zur Aufarbeitung sexuellen Kindesmissbrauchs (2019c): Rechte und Pflichten: Aufarbeitungsprozesse in Institutionen. Empfehlungen zur Aufarbeitung sexuellen Kindesmissbrauchs, 20.12., einzusehe unter https:// www.aufarbeitungskommission.de/wp-content/uploads/2019/12/EmpfehlungenAufarbeitung-sexuellen-Kindesmissbauchs_Aufarbeitungskommission-2019.pdf, zuletzt aufgerufen am 8.11.2O2O.

Wagner, Doris (2019): Spiritueller Missbrauch in der katholischen Kirche. Freiburg: Herder.

Wright, Katie (2017): Remaking Collective Knowledge: An Analysis of the Complex and Multiple Effects of Inquiries into Historical Institutional Child Abuse, in: Child Abuse \& Neglect 74, 10-22. 